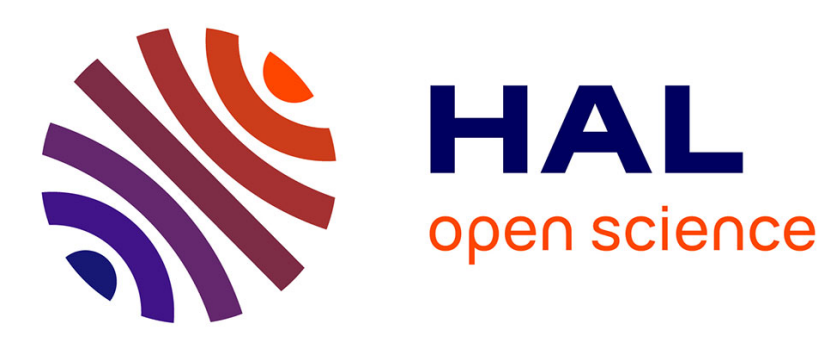

\title{
Entre la rue résidentielle et le boulevard : le cas des soi de Bangkok
}

Eric Charmes

\section{To cite this version:}

Eric Charmes. Entre la rue résidentielle et le boulevard: le cas des soi de Bangkok. Flux - Cahiers scientifiques internationaux Réseaux et territoires, 1998, 34, pp.21-32. halshs-00109857

\section{HAL Id: halshs-00109857 https://shs.hal.science/halshs-00109857}

Submitted on 21 Feb 2007

HAL is a multi-disciplinary open access archive for the deposit and dissemination of scientific research documents, whether they are published or not. The documents may come from teaching and research institutions in France or abroad, or from public or private research centers.
L'archive ouverte pluridisciplinaire HAL, est destinée au dépôt et à la diffusion de documents scientifiques de niveau recherche, publiés ou non, émanant des établissements d'enseignement et de recherche français ou étrangers, des laboratoires publics ou privés. 
- FLUX

$n^{\circ} 34$

Octobre-Décembre

1998

pp. $21-32$
Entre la rue résidentielle et le boulevard : le cas des soi de Bangkok*

ÉRIC Charmes
Éric CHARMES est doctorant à l'Institut français d'urbanisme. Il est membre du département CNRS Théorie des mutations urbaines (UMR 7543). Ses recherches, menées sous la direction de M. Goldblum, portent sur l'interaction des différents usages de la rue. Il s'agit notamment, à partir du cas des soi de Bangkok, de repérer les conflits et les synergies possibles dans l'utilisation de la rue comme outil de valorisation foncière, comme canal de flux circulatoires et comme espace résidentiel ou commercial.
$\mathrm{P}$ OUR LES SPÉCIALISTES de la gestion du trafic, les voies se divisent en quatre grandes catégories, à savoir, par ordre d'importance pour la circulation : les voies de desserte, les voies de distribution, les boulevards et les autoroutes 1 . L'un des objectifs de cette hiérarchisation est d'éviter la multiplication des intersections entre les voies locales et les grands axes de circulation. Les carrefours sont en effet autant de facteurs de ralentissement du trafic et l'une des principales options qui s'offrent aux techniciens pour réduire leur nombre est de créer des voies intermédiaires, distribuant le trafic venu des grandes artères vers les voies de desserte et réciproquement.

Parce qu'elles mêlent usuellement fonction circulatoire et fonction résidentielle, ces voies dites « de distribution » constituent l'une des principales pierres d'achoppement des débats urbanistiques contemporains. Ainsi, si l'on s'en tenait aux modèles proposés par les urbanistes, ces rues n'existeraient pas : cas des voies autoroutières mis à part, la trame viaire des agglomérations urbaines serait restreinte à des rues de quartier et à des boulevards commerçants d'où les habitations seraient exclues. Ces conceptions s'enracinent dans une tradition maintenant ancienne, établie sur le constat d'une opposition entre les besoins de la vie locale et ceux du trafic. En témoigne le fait que la plupart des urbanistes considèrent comme acquis que l'impasse est le meilleur choix pour la localisation d'une résidence.

Comme on va le voir cependant, les transformations d'impasses en voies traversantes en cours à Bangkok contredisent les présupposés qui sous-tendent ces idées. Certes, dans la capitale thaillandaise comme ailleurs, la pollution et le bruit engendrés par le trafic ne sont appréciés de personne, mais les gens savent aussi que ces nuisances sont indissociables d'effets plutôt positifs, tels que l'amélioration de l'accessibilité ou que la possibilité de développer une activité commerciale. Mieux, dans certaines situations économiques et sociales, des riverains peuvent considérer que ces effets positifs compensent largement les désagréments du passage du trafic devant leur résidence.

Même s'il faut se garder des comparaisons hâtives, le détour par le cas des soi (les rues; lire soï) de Bangkok suggère ainsi que les voies de distribution ne

* Cet article reprend certaines conclusions d'une recherche réalisée par l'auteur pour le compte du secrétariat permanent du Plan urbain (voir É. Charmes, Les soi du superbloc de Lad Phrao - De la voie de lotissement à la voiè de liaison : les valeurs de l'espace viaire, Paris, Ministère de l'Aménagement du territoire, de l'équipement et des transports, Laboratoire Théorie des mutations urbaines, 1997, 184 p.). 
sont pas les foyers pathogènes que les urbanistes voient souvent en elles. Loin d'être opposés, les besoins de la vie résidentielle et ceux du trafic peuvent se combiner de diverses manières. La différenciation des rues ne tire donc pas simplement sa légitimité du fait qu'elle participe à la fluidification du trafic comme on le dit souvent : elle répond aussi à la diversité des articulations possibles entre les usages circulatoires et les usages résidentiels ou commerciaux de l'espace viaire.

\section{L'IMPASSE : LA VOIE RÉSIDENTIELLE PAR EXCELLENCE ?}

\section{NOTES SUR LE STATUT DE L'IMPASSE DANS L'URBANISME VIAIRE}

Depuis les grandes enquêtes d'opinion menées dans les années 1970 dans différents pays occidentaux, on sait que le trafic automobile est, au delà d'un certain stade que Donald Appleyard a fixé à 2000 véhicules par jour, mal accepté par les riverains ${ }^{2}$. L'impact du trafic ne se limite pas aux désagréments d'ordre physiologique occasionnés par le bruit, la pollution et les vibrations ; celuici s'étend aux relations sociales et au psychisme des riverains. Ainsi, les relations de voisinage, de même que l'attachement et le sentiment d'appartenance à la rue sont beaucoup plus faibles dans les voies très fréquentées, que dans les rues résidentielles les plus calmes ${ }^{3}$.

Les urbanistes n'ont cependant pas attendu les résultats de ces enquêtes quantitatives pour mettre en avant la qualité résidentielle des impasses qui, fermées à tout trafic extérieur, restent éloignées du bruit et de la pollution. Elles ont ainsi été utilisées dès les premières étapes du développement de l'usage de l'automobile pour tenter de concilier mobilité et enracinement. Les premières propositions prirent forme à la fin des années 1920 dans le New Jersey, quand Clarence Stein et Henry Wright apportèrent la touche finale à un projet d'aménagement pour le lotissement de Radburn. En partie inspirés par les travaux de Raymond Unwinn, les deux architectes prônaient principalement une séparation complète de l'automobile et du piéton au moyen d'une spécialisation des voies. Ainsi, dans leur projet, les rues permettant l'accès motorisé aux habitations étaient essentiellement des impasses (voir Figures 1 et 2). Celles-ci desservaient 15 ou 20 maisons individuelles et, du côté opposé à celui donnant sur la rue,
Figure 1. Plan de Radburn, New Jersey, Clarence Stein \& Henry Wright, 1929 (in C. S. Stein, Towards New Towns for America, Cambridge, MIT Press, 1966, p. 43 ; cité par C.L. Girling \& K.I. Helphand, Yard, Street, Park, p. 61).

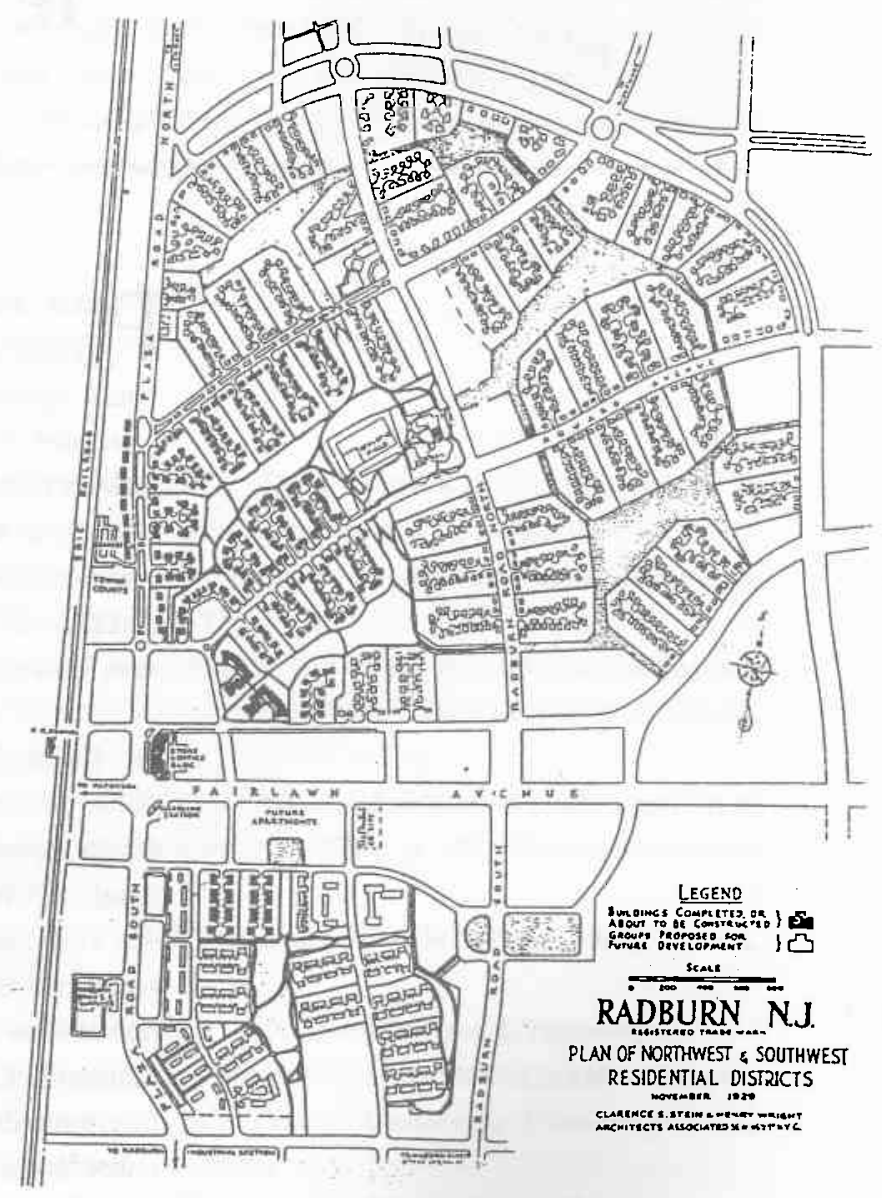

chaque résidence ouvrait sur des espaces verts et sur des circulations piétonnières censées constituer l'épine dorsale du lotissement. Groupées par dizaines ou par douzaines, les impasses résidentielles formaient ce que Stein appelait des superblocs. Le trafic de transit était rejeté à l'extérieur de ces derniers, sur les voies artérielles qui les entouraient ${ }^{4}$. Comme Colin Buchanan le reconnut luimême ${ }^{5}$, ces idées furent reprises quasiment à l'identique une trentaine d'années plus tard dans le rapport qui porte son nom et qui constitua l'acte de naissance des fameuses « aires environnementales » (voir Figure 3 ).

Bien évidemment, les modèles développés par ces urbanistes ne se sont pas incarnés tels quels dans la réalité. Dès le départ, Clarence Stein lui-même s'attendait à ce que la séparation fonctionnelle ne soit pas totale. Il savait 
Figure 2. Cul-de-sac, Radburn, New Jersey (in C. S. Stein, Towards New Towns for America, Cambridge, MIT Press, 1966, p. 43 ; cité par C.L. Girling \& K.I. Helphand, Yard, Street, Park, p. 61).

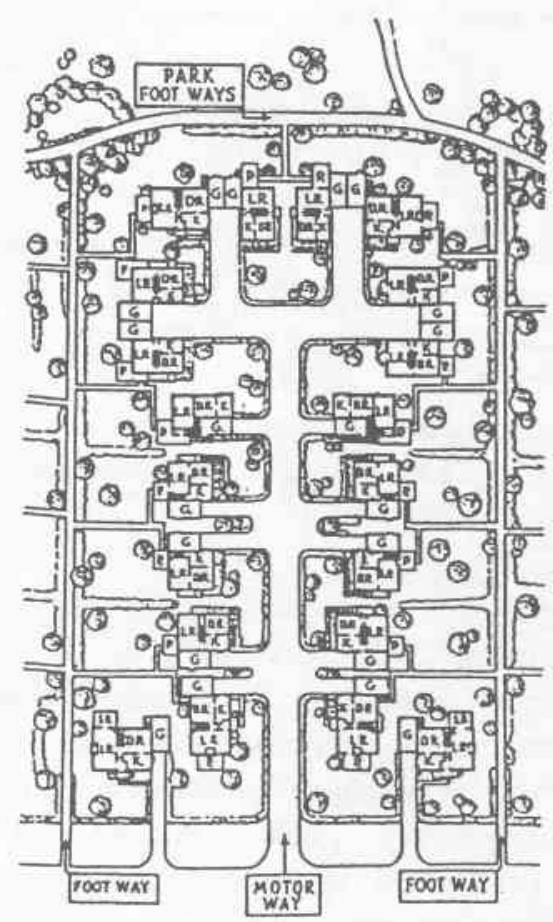

en effet que, pour certaines activités (le vélo et les jeux de balle notamment), les enfants prisent particulièrement un revêtement en dur. Dans son esprit, cantonner les habitations dans des impasses était d'ailleurs un moyen de faire des rues résidentielles des terrains de jeu relativement sûrs pour les enfants ${ }^{6}$. Il n'avait cependant pas prévu que, dans la plupart des lotissements américains, les rues en boucle et les impasses deviendraient des lieux de sociabilité beaucoup plus actifs que les allées et que les parcs spécialement prévus à cet effet ${ }^{7}$. Surtout, il ne s'attendait probablement pas à ce que le plan qu'il avait dessiné pour Radburn s'imposerait, via le rapport Buchanan, comme une des références principales de l'urbanisme viaire contemporain dominé, du moins dans les pays développés, par les woonerven et autres "rues partagées », où véhicules motorisés, passants et enfants sont censés coha- biter harmonieusement sur l'ensemble de l'emprise de la rue $^{8}$. Bref, contrairement à ce qu'ont pu espérer certains urbanistes fonctionnalistes, la spécialisation des voies n'a jamais rimé avec monofonctionnalité et les praticiens ont dû prendre acte de l'indéfectibilité du lien entre circulation automobile et espaces de vie locaux.

Il reste que, dans l'idéal des urbanistes, aujourd'hui comme dans l'Entre-deux-guerres, la rue résidentielle demeure profondément associée à la figure de l'impasse et donc opposée au trafic. La voiture n'est admise que par défaut au cœur de la vie résidentielle et, au motif qu'elle ne lui apporte rien, la circulation de transit est rejetée sur des axes extérieurs au quartier. À Bangkok comme ailleurs, cette opposition trouve sa légitimité dans l'attitude des riverains face aux nuisances engendrées par la circulation automobile.

\section{LE RAPPORT AUX NUISANCES DU TRAFIC À BANGKOK}

Sans disposer des résultats d'enquêtes aussi massives que celles réalisées aux États-Unis et en Grande Bretagne dans les années 1970, on peut affirmer sans grand risque d'erreur que les nuisances du trafic sont peu appréciées par les habitants de la capitale thaïlandaise.

La quasi ubiquité des ralentisseurs dans les rues résidentielles de Bangkok démontre d'ailleurs aussi bien que n'importe quelle enquête que, si les Bangkokiens témoignent pour l'observateur étranger d'une résistance peu commune aux odeurs, à la poussière et aux bruits extérieurs, le trafic n'en est pas moins pour eux une nuisance qu'ils ne se sont pas totalement résignés à accepter. Interrogés, élus locaux, responsables municipaux et gérants de lotissement ${ }^{9}$ confirment tous cette impression et disent recevoir régulièrement des plaintes de la part de riverains incommodés par le passage de chauffards. La demande de pose de ralentisseurs est telle que, si elle était entièrement suivie, certaines rues seraient impraticables. Cette focalisation sur la vitesse des véhicules s'explique sans doute par le fait qu'il existe, avec les « gendarmes couchés ", un moyen simple et peu coûteux de réduire ce problème, ce qui n'est guère le cas avec les autres effets négatifs du trafic, tels que le bruit, les vibrations ou la pollution. Il convient par ailleurs de souligner que la plupart des soi de Bangkok ne disposent pas de trottoirs, alors même qu'ils peuvent supporter un trafic important 
Figure 3. Le principe d'aménagement de Radburn vu par Colin Buchanan (in C. Buchanan et al., Traffic in Towns, p. 47).
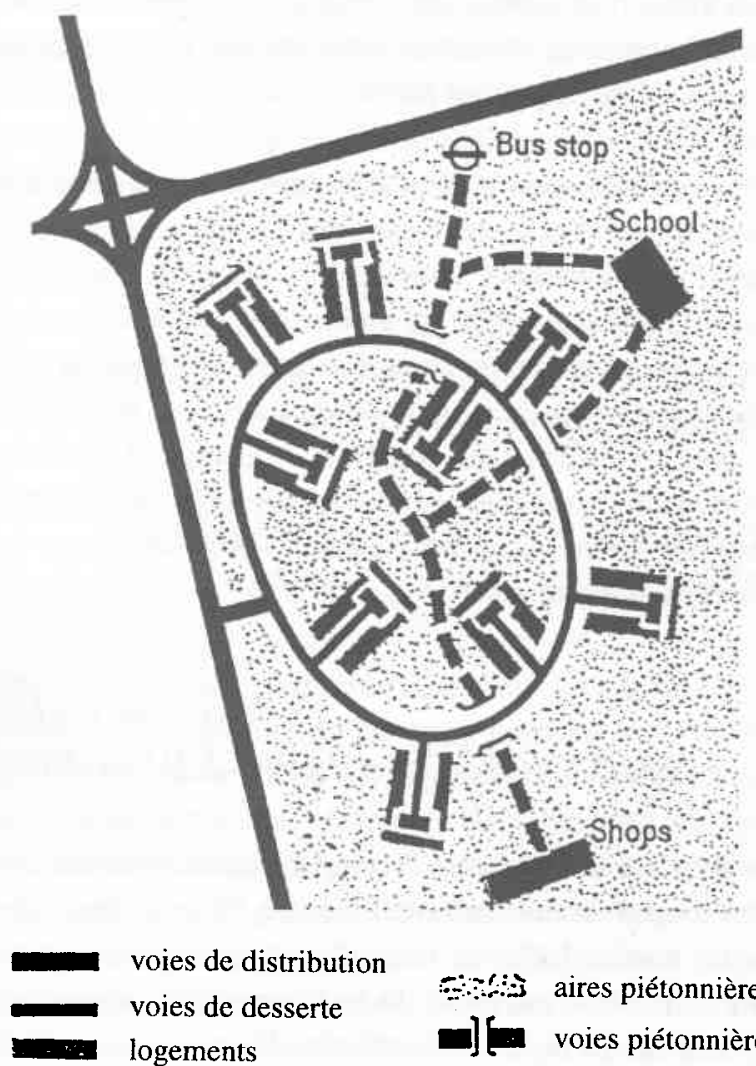

voies de distribution voies de desserte logements

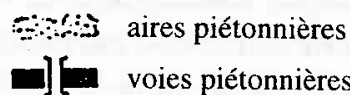

(voir Photographie 1). Le piéton se trouve donc sans protection face au flot des véhicules motorisés et, surtout, face aux motos qui, lors d'encombrements, s'infiltrent à travers le moindre espace laissé libre. Le problème est particulièrement aigu dans les lotissements les moins huppés, où les enfants sont poussés dans la rue par la chaleur et par l'exiguité des logements.

Ainsi, dans une impasse intégrée moins d'un an auparavant à un itinéraire de liaison, le soi Charœn Phon (voir Photographie 2), quasiment tous les riverains disent ${ }^{10}$ ressentir à des degrés divers une gêne. Seule une femme, travaillant comme vendeuse de rue et donc probablement accoutumée aux nuisances de la circulation, affirme ne pas être dérangée et percevoir au contraire le bruit des voitures comme une musique d'accompagnement. Les autres se déclarent d'une manière ou d'une autre perturbés par le passage du trafic : ainsi, $20 \%$ des foyers interrogés disent être affectés par la pollution ; $40 \%$ affirment être dérangés par le bruit ; $40 \%$ avouent être gênés par les vibrations du sol provoquées par le passage de poids lourds et, surtout, $73 \%$ considèrent que les véhicules roulent à une vitesse trop élevée ou sont dangereux pour les enfants. Le contentement de la vendeuse de rue ellemême est d'ailleurs tempéré par des craintes concernant la vitesse des véhicules.

L'attention portée aux chauffards est probablement exacerbée par la largeur du soi (l'emprise du revêtement de béton armé avoisine les 12 mètres), par son caractère rectiligne (près de 400 mètres en ligne droite) et, surtout, par l'irrégularité du trafic, relativement faible en dehors des heures de pointe. En effet, ainsi que l'a déjà observé Donald Appleyard, un flot continu de véhicules maintient constamment l'attention en éveil, alors qu'un chauffard survenant dans une rue peu fréquentée peut surprendre et provoquer un accident, particulièrement lorsque des enfants utilisent la rue comme aire de jeux ${ }^{11}$. Parallèlement, bruit, pollution et vibrations sont des problèmes moins préoccupants dans une rue peu fréquentée que dans une rue très passante.

Une telle série d'entretiens ne peut certes pas prétendre à une véritable représentativité à l'échelle de l'agglomération de Bangkok. On retrouve cependant très exactement dans les réponses des riverains du soi Charæn Phon les principaux sujets de plaintes concernant le trafic relevés au début des années 1970 à San Francisco par Donald Appleyard et son équipe ${ }^{12}$, ainsi que les principales préoccupations qui guident encore aujourd'hui l'aménagement des voies dans les pays développés, notamment la sécurité 13 . Cette convergence de données est d'autant plus significative que, dans le cas du soi Charœn Phon, les différents sujets de récriminations ont été évoqués spontanément et non en réaction à une liste des nuisances présumées du trafic. Ceci permet donc de conjecturer sans grand risque d'erreur que les Thaillandais ont un rapport au trafic relativement commun.

\section{LA TRANSFORMATION DES IMPASSES EN VOIES TRAVERSANTES : UNE HÉRÉSIE?}

On peut donc légitimement supposer qu'à Bangkok comme ailleurs, la transformation d'une impasse en voie traversante est vécue fortement négativement par les riverains. C'est d'ailleurs sans plus de considérations ce qu'affirme Donald Appleyard dans un texte aux accents 


\section{Charmes - Entre la rue résidentielle et le boulevard à Bangkok}

Photographie 1. Entrée du soi Aphaphirom, près du boulevard Rachadapisek.

Ainsi que l'indique le panneau situé en haut à gauche sur ce cliché, ce soi résidentiel a été transformé en voie de liaison par la municipalité. Comme la plupart des soi de ce type, cette voie n'est guère adaptée à cet usage, d'autant qu'elle est ouverte à la circulation dans les deux sens. Seuls les poteaux électriques viennent définir un espace piétonnier.

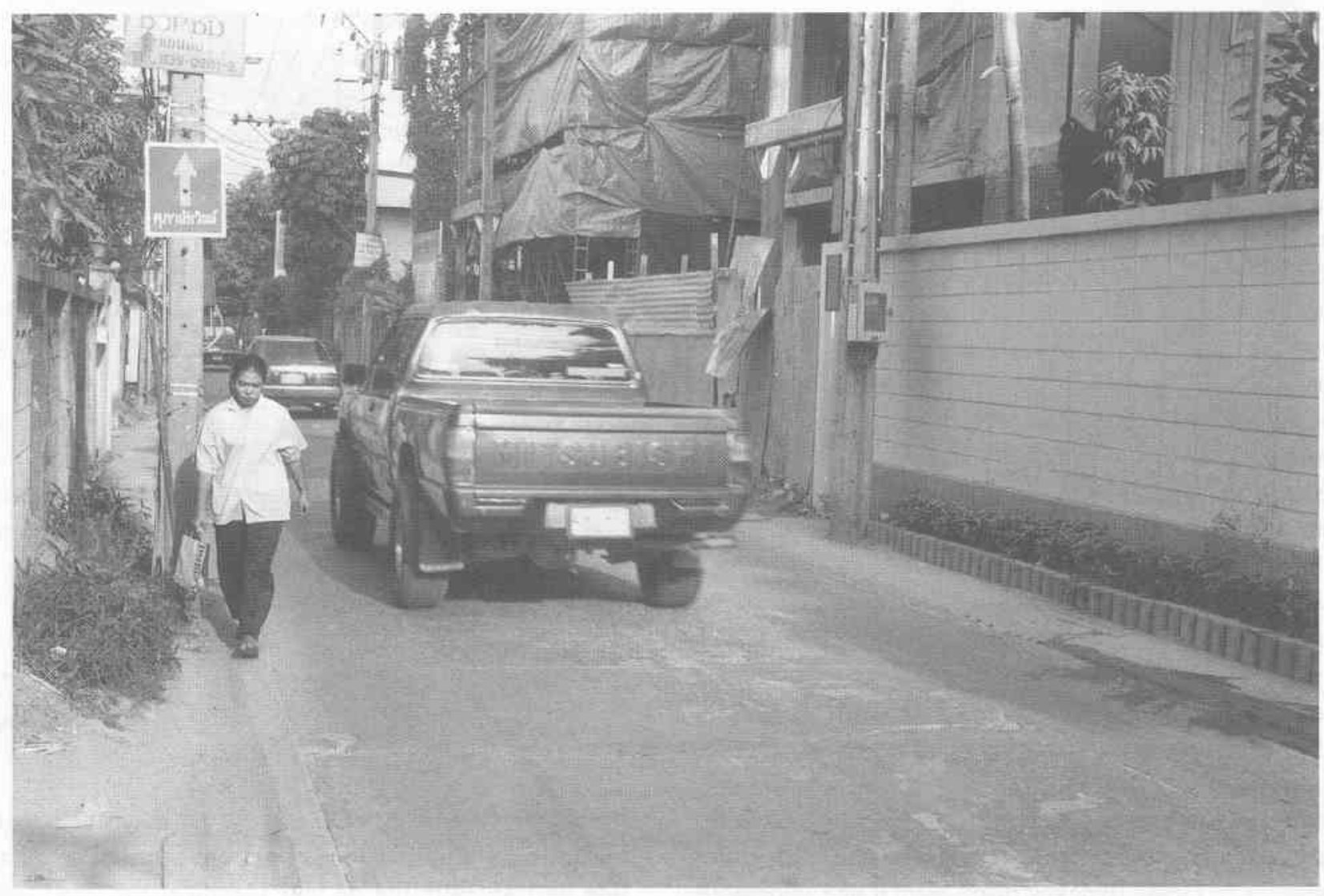

prophétiques intitulé : « Les rues peuvent tuer les villes : Tiers Monde prend garde ! ». Appelant les villes en développement à ne pas reproduire les erreurs des villes occidentales et, plus précisément, à ne pas laisser l'automobile s'approprier l'espace viaire, Donald Appleyard cite en exemple les villes d'Ahmedabad et de Bangkok, dont les pols et les soi sans issue sont autant d'espaces protégés des nuisances du trafic ${ }^{14}$. Pour lui, ces voies constituent un patrimoine précieux, équivalent organique des aires environnementales de Colin Buchanan et des woonerven néerlandaises.

Dans la pensée du chercheur américain, les inconvénients de la transformation des voies sans issue en voies traversantes ne se réduisent cependant pas à une perte patrimoniale. En effet, ce processus aboutit à la création de voies hybrides, qui cumulent des fonctions liées à la présence d'habitations riveraines avec d'autres, liées au passage d'un trafic extérieur au quartier. Or, comme on peut le voir sur la Photographie 1, cette situation est source de conflits difficiles à gérer.

La conclusion logique d'un tel constat est qu'il vaut mieux éviter le développement des voies de distribution et articuler le système viaire autour de deux types de voies : les impasses résidentielles et les boulevards urbains, où les activités commerciales peuvent se développer en symbiose avec le trafic. Aujourd'hui, cette ligne de pensée, dont Donald Appleyard n'est que l'un des représentants les plus éminents, n'est pas loin d'être victorieuse. Elle est en tous cas l'une des plus influente des urbanismes européen et nord-américain ${ }^{15}$. Partant, la Thailande puisant une bonne part de ses modèles de développement dans l'expérience occidentale, on peut à bon droit s'étonner de ce que sa capitale persiste à transformer des impasses en voies traversantes. 


\section{FLUX n³4 Octobre-Décembre 1998}

\section{L'IMPASSE À L'ÉPREUVE DE L'ACCESSIBILITÉ ET DU DÉVELOPPEMENT COMMERCIAL}

On peut certes douter de la pertinence d'une interrogation des pratiques de pays développés par le biais de celles d'un pays en développement. De fait, les conditions de circulation calamiteuses qui règnent à Bangkok, comme dans la plupart des capitales du Tiers Monde, induisent une priorité de l'amélioration des réseaux de transports sur la protection du cadre de vie des riverains, à l'inverse des tendances actuellement dominantes dans les pays développés. Sur ce point, les pouvoirs publics thaîlandais sont en accord avec leurs administrés : toutes les enquêtes montrent que les encombrements sont perçus par les Bangkokiens comme le principal problème affectant leur vie quotidienne, loin même devant la question du logement ${ }^{16}$. Or, l'une des principales causes de ces difficultés de circulation est le surnombre des impasses, lié tant au manque d'encadrement des activités immobilières et foncières privées, qu'au grand nombre de canaux qui bloquent les voies dans leur expansion. En périphérie, des ensembles de plusieurs dizaines de kilomètres carrés sont ainsi desservis par un entrelacs de voies qui ne laisse guère d'autres possibilités à l'automobiliste que d'emprunter les quelques grandes artères construites par les pouvoirs publics (voir Figure 4, p. 30). Ces dernières sont donc constamment engorgées, malgré un taux de motorisation relativement faible (en 1994, seuls $22 \%$ des ménages du Grand Bangkok disposaient d'une automobile ou d'un véhicule utilitaire). Dans un tel contexte, on comprend aisément que la création de voies distribuant le trafic des grands axes urbains vers les voies de desserte s'impose comme une nécessité première. On peut donc penser que la transformation d'impasses en voies traversantes n'est que la conséquence inévitable de contraintes économiques et sociales propres à la ville en développement qu'est Bangkok et qu'elle ne retire rien de sa portée critique à la modélisation dichotomique de l'espace viaire.

Une remise en cause beaucoup moins superficielle de ce modèle est toutefois suggérée par l'absence de volonté individuelle de rejeter sur les autres le poids des transformations indispensables de la trame viaire, sur le modèle du fameux syndrome « NIMBY » (de l'anglais not in my backyard, que l'on peut traduire approximativement par « pas dans mon jardin »). Ainsi, il arrive que les riverains de soi sans issue soient eux-mêmes à l'origine de leur conversion en voie traversante. Mieux, même si l'initiati- ve d'une telle transformation ne leur revient pas, il n'est pas rare que les riverains s'en déclarent satisfaits. Or, de telles attitudes s'opposent à l'hypothèse qui sous-tend la promotion de l'impasse comme idéal de la rue résidentielle, à savoir que les riverains d'une rue préfèrent l'absence de trafic à sa présence. En fait, les comportements et les jugements observés à Bangkok révèlent d'une part, que cette hypothèse n'est valable que « toutes choses égales par ailleurs », et d'autre part, que les choses ne sont dans la réalité pas toujours égales par ailleurs. Ainsi, les effets externes du trafic ne se limitent pas à des nuisances : les voies où la circulation se concentre sont aussi les plus accessibles et sont souvent le siège d'un important développement commercial, dû en grande partie à l'effet vitrine engendré par le trafic. Étant donné que l'on ne sait pas encore réaliser de véhicules parfaitement silencieux et non polluants, ces effets bénéfiques du trafic sont indissociables des inconvénients mentionnés précédemment. Les riverains le savent et pèsent, comme on va le voir, le pour et le contre avant de porter un jugement sur la transformation de leur impasse en voie traversante.

\section{L'ACCESSIBILITÉ : RÉSEAU MAILLÉ CONTRE RÉSEAU ARBORESCENT}

Nous avons été confronté à Bangkok à plusieurs cas où les habitants d'un lotissement ont fait primer l'amélioration de l'accessibilité de leur logement sur toute autre considération. Dans certains cas, comme dans celui des lotissements (muban en thai) Prachakon Thai et Suanson, les comités des copropriétaires sont allés jusqu'à réclamer eux-mêmes l'ouverture d'un deuxième point d'accès et donc l'ouverture de leur lotissement au trafic de transit ${ }^{17}$. Les raisons de cette attitude sont relativement simples ${ }^{18}$ : l'entrée principale de chacun de ces muban débouche sur une avenue extrêmement congestionnée. D'où l'attrait de la possibilité d'emprunter d'autres itinéraires ; attrait tel que, pour les habitants du lotissement, l'ouverture d'une deuxième entrée compensait largement les nuisances du trafic et, plus encore, la peur de l'augmentation de l'insécurité (crainte très fréquemment mentionnée par les riverains et par les gérants de lotissement parmi les inconvénients de la transformation d'une impasse en voie traversante).

Le cas précédemment évoqué du muban Charæn Phon est encore plus probant, puisque la demande de 
Photographie 2. Le soi Charæn Phon.

Sa grande largeur incite les automobilistes à aller vite et fait de cet espace un lieu dangereux. Il est à noter que cette largeur excessive résulte de normes concernant la subdivision foncière, mises en place en Thaïlande à partir des années 1970.

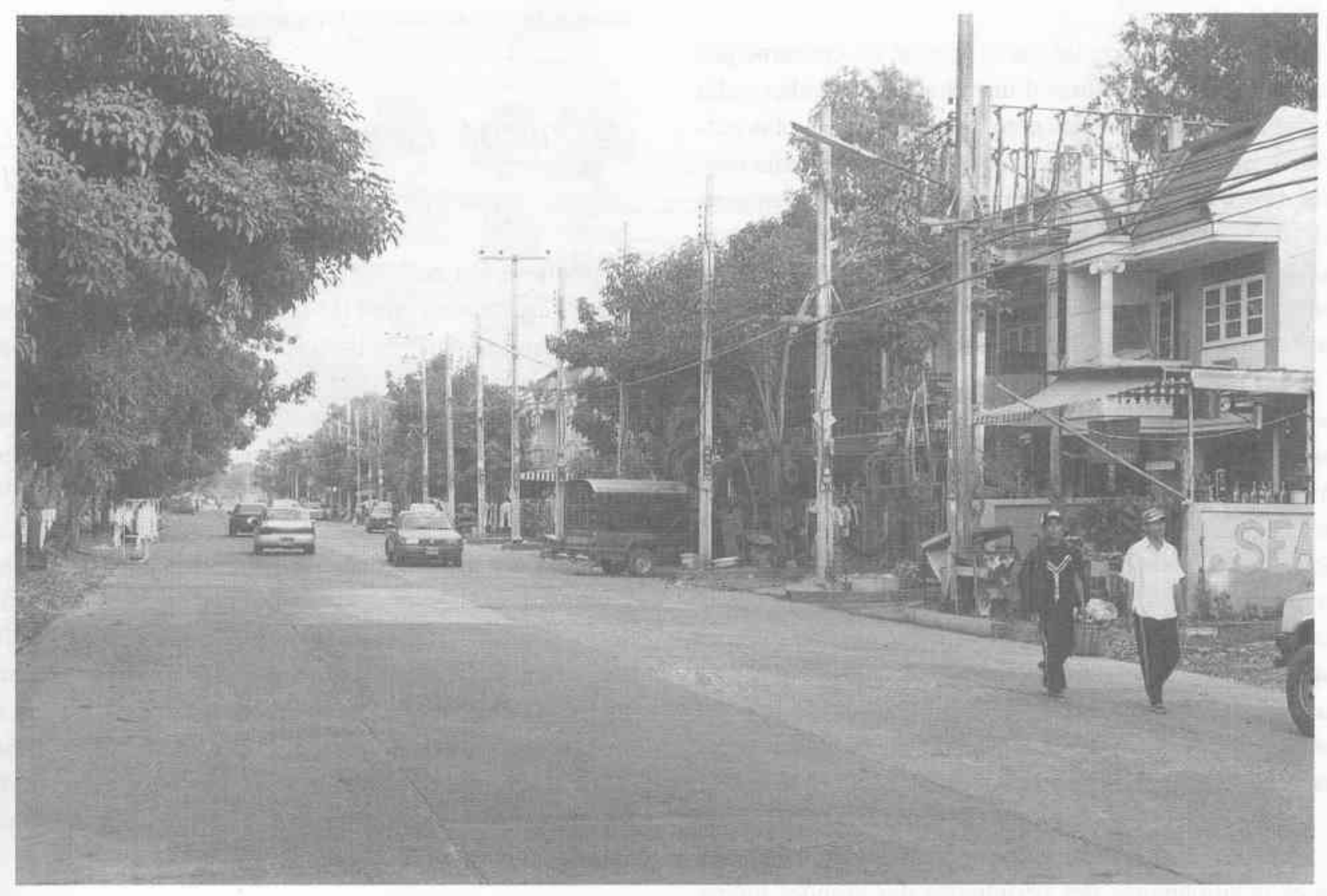

création d'un itinéraire de liaison à travers ce lotissement a principalement émané, non pas des résidants euxmêmes, mais d'habitants des quartiers alentours qui souhaitaient disposer d'un accès plus direct à une école située à proximité. De plus, la décision d'ouvrir le lotissement au trafic extérieur a été prise par le promoteur, sans consultation préalable des copropriétaires. Il n'empêche, la conclusion reste la même. La quasi totalité des riverains du soi du muban Charcen Phon ouvert à la circulation publique s'estiment certes gênés par le trafic, comme on l'a vu, mais dans le même temps une large majorité d'entre eux (75\% des personnes interrogées) considèrent que cette gêne est compensée par l'accessibilité qu'apporte une telle transformation.

Ce résultat est d'autant plus remarquable que, la voie étant ouverte depuis peu au trafic, il ne peut être attribué à une quelconque résignation des habitants à la mauvaise qualité de leur environnement - effet d'adaptation envi- ronnementale dont Donald Appleyard a souligné l'ampleur dans ses observations sur l'opinion des riverains des rues les plus bruyantes et les plus polluées de San Francisco ${ }^{19}$. De plus, les maisons en bande du soi du muban Charcen Phon ouvert au trafic de transit donnent quasiment directement sur la rue : leur façade n'en est séparée que par un modeste espace bétonné dans lequel un véhicule de petite taille peut à peine stationner (voir Photographie 2). Avant l'ouverture de l'itinéraire de liaison, l'espace viaire était d'ailleurs largement utilisé comme extension du logement, comme en témoignent les panneaux de basket encore accrochés à quelques portails, ainsi que les tables et les chaises installées sur le bord de la rue pour accueillir d'éventuels visiteurs. Le trafic de transit, nettement plus important que celui qui traverse le muban Prachakon Thai, se trouve donc réellement en compétition avec des usages locaux de la rue : de fait, aujourd'hui, les enfants jouent plus rarement au basket devant chez eux, leurs parents le leur interdisant de peur 
qu'ils n'aient un accident. Cette perte, réelle et consciente, de possibilités d'usages locaux de l'espace viaire souligne clairement à quel point l'accès peut être un enjeu important.

Cette amélioration de l'accessibilité ne concerne pas seulement les propriétaires d'un véhicule particulier : elle touche aussi les personnes n'en disposant pas et plus particulièrement les personnes âgées. Ainsi, une femme résidant dans l'un des sous-soi du muban Charœn Phon nous a déclaré regretter que la nouvelle voie de liaison ne passe pas devant chez elle ${ }^{20}$. Elle doit en effet marcher quelques centaines de mètres pour atteindre le soi principal du lotissement et avoir une chance de pouvoir arrêter un taxi. Il y a bien quelques véhicules de transport en commun (songthaew) qui passent devant chez elle, mais ils ne le font qu'aux heures de pointe, le matin et le soir. Des chauffeurs de taxi-moto sont eux présents toute la journée, mais elle juge ce mode de transport trop inconfortable. La situation s'est toutefois améliorée avec l'ouverture de la voie de liaison puisqu'auparavant, elle devait marcher jusqu'à l'entrée du lotissement, soit une distance supplémentaire d'environ 350 mètres. Les difficultés évoquées par cette personne âgée sont bien évidemment les mêmes pour une personne chargée de lourds colis ou pour une femme enceinte.

On retrouve ici une critique souvent adressée aux zones pavillonnaires des périphéries des grandes métropoles : le calme et la tranquillité des rues qui desservent ces quartiers n'effacent pas le fait que ceux-ci sont de véritables prisons pour les populations non motorisées, adolescents et personnes handicapées ou âgées notamment $^{21}$. Pour ces dernières, la séparation stricte de la valeur territoriale et de la valeur locale de l'espace viaire n'est pas viable. C'est d'ailleurs un constat similaire qui nourrit la critique actuelle de la desserte des Grands Ensembles ou encore le courant, parfois abusivement dit « néo-traditionnel », emmené aux États-Unis par Andres Duany et Elisabeth Plater-Zyberk ${ }^{22}$. De plus en plus influents, ces urbanistes proposent de supprimer les impasses et les voies en boucles, qui dominent tant le paysage des lotissements de la périphérie des métropoles américaines que celui des Grands Ensembles français, pour les remplacer par des voies traversantes bordées de maisons mitoyennes, suivant un modèle proche de celui fourni par le centre des villes traditionnelles. On peut ainsi lire dans une publication récente du CERTU : «Un des objectifs habituels de l'aménagement des quartiers réservés à l'habitat consiste à les préserver de la circulation de passage pour éviter les nuisances. Lorsqu'on applique ce principe à des unités urbaines qui comptent plus de 5000 habitants, on fabrique des isolats, calmes, mais sans vie urbaine et éloignés de tout $\gg 23$.

\section{LE DÉVELOPPEMENT COMMERCIAL : L'APPORT DU TRAFIC}

L'amélioration de l'accessibilité n'est toutefois pas la seule raison pour laquelle les riverains du soi Charœn Phon sont satisfaits de l'ouverture de leur environnement résidentiel au trafic extérieur. Les opportunités de développement commercial offertes par cette transformation ont aussi joué un rôle (voir Photographie 3). De fait, tout cartogramme de la répartition des commerces en milieu urbain le montre, commerce et trafic se nourrissent l'un l'autre $^{24}$. La capitale thaïlandaise ne fait pas exception à cette règle : les rues les plus populaires et les plus animées sont aussi celles qui supportent le trafic le plus important (à l'exclusion encore une fois des autoroutes urbaines). Pour les échoppes et les restaurants en plein air qui s'alignent sur les trottoirs, le bénéfice de l'effet vitrine semble largement compenser les nuisances d'un trafic pourtant souvent tumultueux et générateur de multiples fumées et poussières.

Les rues à vocation résidentielle de Bangkok peuvent aussi tirer parti de cet effet d'entraînement du trafic sur les activités commerciales. Dans cette ville en développement, la mobilité est en effet relativement faible. En 1989, les hommes effectuaient en moyenne 2,71 déplacements par jour et les femmes seulement 1,77, contre respectivement 3,3 et 3,1 en France ${ }^{25}$. Même à basse densité par conséquent, l'ouverture d'un commerce de proximité peut être viable. Ainsi, dans les lotissements destinés aux classes moyennes, les rues bruissent tout au long de la journée d'une activité incessante engendrée tant par les diverses habitations converties en bureaux ou en ateliers que par les épiceries, les restaurants ou les salons de beauté installés au rez-de-chaussée de nombreux pavillons ${ }^{26}$. Pour les fonctionnaires, les employés ou les artisans qui vivent là, ces activités commerciales permettent de disposer de ressources d'appoint ${ }^{27}$. Elles présentent en outre l'avantage de pouvoir être conciliées avec la tenue d'un ménage : une mère de famille, notamment, peut gérer un petit commerce au pied de son domicile tout en conti- 
Photographie 3. L'avenir possible du soi Charœn Phon. Il s'agit du soi Nakniwat, près de son intersection avec le soi Sahakon 3.

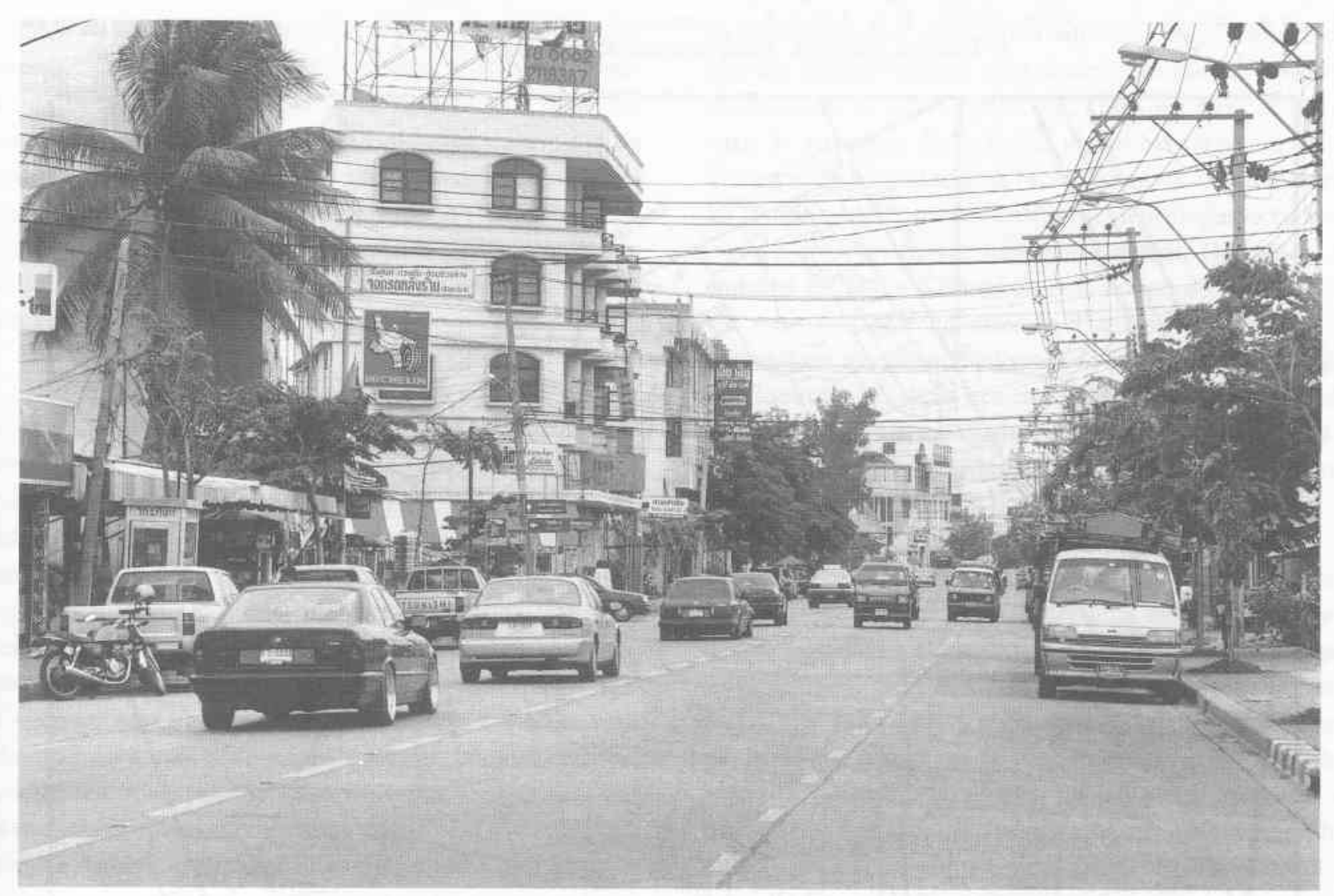

nuant à s'occuper de ses enfants. Ces demiers peuvent d'ailleurs l'assister dans son travail.

Pour ces familles, un accroissement du trafic passant devant leur domicile est synonyme d'augmentation du nombre de clients potentiels. Ainsi, les quelques commerçants qui étaient implantés au bord du soi Charæn Phon avant son ouverture au trafic de transit suscitent l'envie de ceux dont les étals n'ont pas la chance de se trouver en bordure de la nouvelle voie de liaison. Plus de la moitié de ces derniers 28 affirment même que, si la possibilité leur en était offerte, ils déménageraient volontiers pour s'installer près de la circulation, d'autant que, la construction du lotissement ayant été abandonnée en cours de route, le nombre de résidants et donc de clients proches n'est pas à la hauteur de leurs espérances.

Il faut toutefois se garder de faire de la répartition des flux circulatoires l'alpha et l'oméga de la distribution des activités commerciales. Celle-ci dépend en réalité de rap- ports complexes, dont l'environnement est une donnée toute aussi essentielle que la desserte ${ }^{29}$. Dans le cas du soi Charœen Phon, le développement des commerces est ainsi moins motivé par le passage du trafic que par la proximité d'une clientèle importante, constituée par les résidants du lotissement. La plupart des échoppes ont d'ailleurs été ouvertes avant la création de l'itinéraire de liaison. Le supplément de clientèle apporté par un accroissement du trafic peut certes être appréciable - et apprécié - il n'en demeure pas moins un critère d'ordre secondaire dans l'évaluation de la viabilité du commerce. Pour preuve, un restaurant de poissons qui s'était installé à l'entrée du muban Charœen Phon peu après son ouverture au trafic de transit, n'est pas parvenu à réunir la clientèle nécessaire et a dû fermer ses portes quelques mois plus tard. La publicité offerte par le trafic n'a apparemment pas été suffisante pour une activité aussi spécialisée.

En tout état de cause, il est peu probable que l'on puisse observer de telles situations dans les banlieues des 
Figure 4. L'entrelacs des voies à l'intérieur du maillage des grandes artères.

Situé à une quinzaine de kilomètres du centre d'affaires, cet ensemble couvre une surface d'environ $72 \mathrm{~km}^{2}$ et abritait 290000 personnes en 1990, sur une population totale urbaine pour la capitale thaillandaise de 6,7 millions de personnes. Le soi Charœn Phon se trouve au coeur de cet ensemble. (Source : R.W. Archer \& N. Topananont, Financing the Construction of Distributor/Collector Roads in Thailand, Bangkok, Asian Institute of Technology, 1995, p. 4A.)

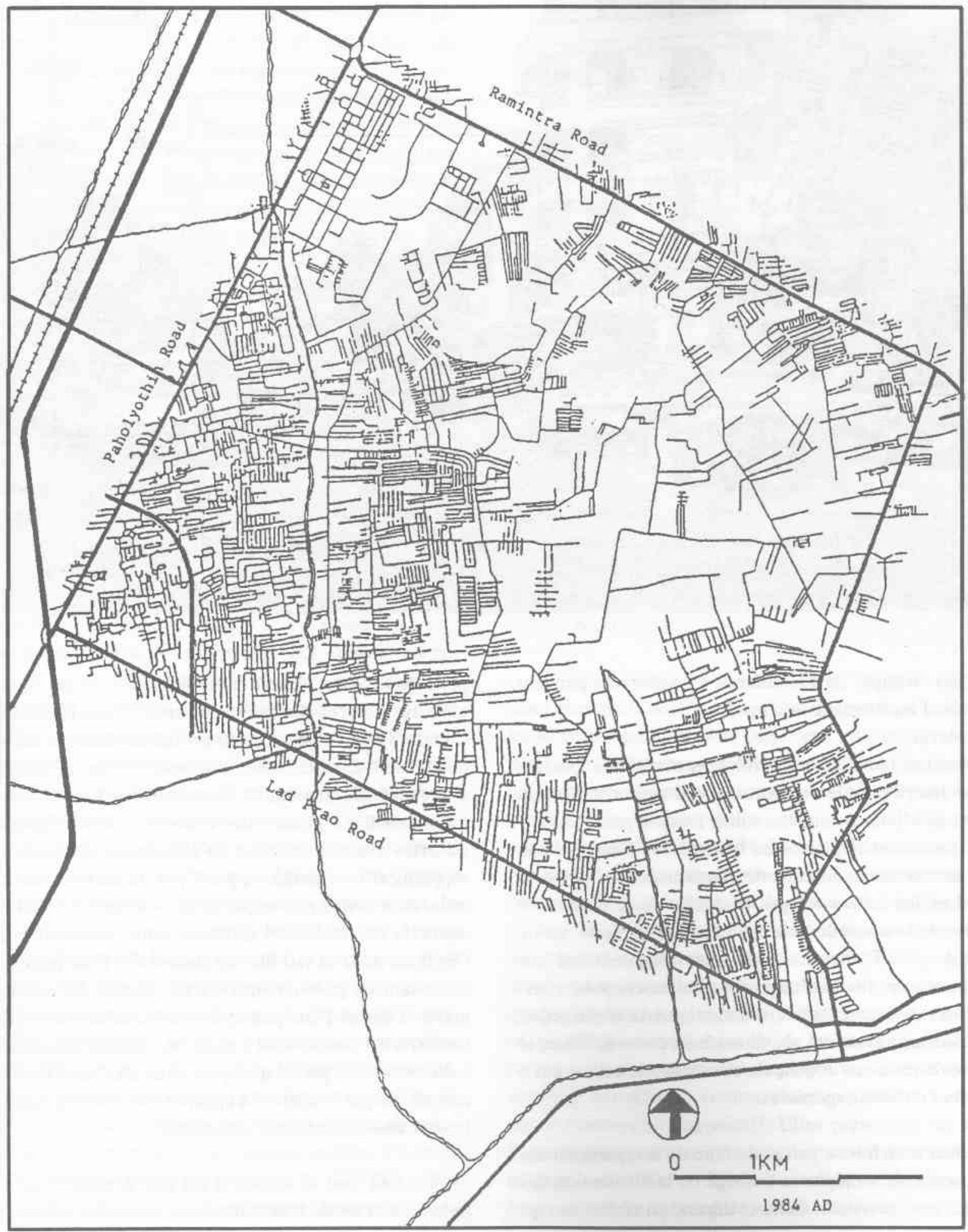




\section{Charmes - Entre la rue résidentielle et le boulevard à Bangkok}

grandes métropoles américaines ou européennes. Les lotissements y ressemblent plutôt aux ensembles résidentiels huppés de la périphérie de Bangkok. Les rues sont calmes en dehors des heures de sortie et de rentrée des bureaux, et les interactions entre voisins tendent à se limiter à des salutations anonymes. La densité plus faible (le nombre de personnes par foyer baisse avec l'élévation du revenu) et surtout, la mobilité plus grande des habitants laissent peu de chances à un commerce d'être viable. Les règles strictes de zonage qui inspirent généralement les règlements de copropriété auraient de toutes façons raison des velléités d'un éventuel entrepreneur.

Eu égard aux débats urbanistiques occidentaux, il n'est toutefois pas inutile d'insister sur le mécanisme mis en évidence ici. Le lien entre trafic et activités commerciales, s'il n'est pas à proprement parler une découverte, est parfois oublié. Actuellement, les débats idéologiques sur la ville sont dominés par la volonté de réduire la place prise par la voiture. L'une des idées les plus discutées est celle, relativement ancienne, de densification ${ }^{30}$. Une idée complémentaire, paradoxale il faut en convenir, serait d'accepter que l'automobile pénètre dans certaines rues résidentielles, non pour y rouler au pas, comme dans les woonerven, mais pour y circuler. De fait, si certains urbanistes américains contemporains s'affirment favorables au plan en damier, c'est non seulement, comme on l'a vu, en raison de la meilleure accessibilité qu'il offre aux habitants, mais aussi parce que les commerces s'implantent plus facilement dans une voie traversante que dans une impasse ${ }^{31}$. Les résidants, à condition bien sûr que le tissu urbain soit assez dense, disposeraient donc avec une plus forte probabilité de la possibilité de faire certains de leurs achats à pied. Ces propos ont une résonance particulière aux États-Unis dans la mesure où il y est courant que, pour chaque achat quotidien, les gens prennent leur voiture pour sortir de leur impasse et rejoindre une voie principale menant à un centre commercial.

\section{CONCLUSION : \\ LA DIFFÉRENCIATION DE LA TRAME VIAIRE}

En résumé, le calme et l'absence de pollution ne s'obtiennent pas « toutes choses égales par ailleurs », mais bien en l'échange d'un certain isolement, tant sur le plan commercial, que sur celui de l'accessibilité. Les riverains des rues de Bangkok en sont parfaitement conscients et sont prêts, en fonction de leur situation, à différents compromis. Bien sûr, ces comportements sont déterminés par un contexte économique et social particulier. Dans les pays occidentaux, la structure de la trame viaire est rarement aussi désordonnée qu'à Bangkok (voir Figure 4). L'intérêt pour les riverains de la transformation de leur impasse en voie traversante s'en trouve diminué d'autant. De même, la mobilité est faible à Bangkok et la notion de « qualité de vie » est loin de rencontrer en Thaïlande l'écho qu'elle trouve dans les pays développés ${ }^{32}$. Toutes différences qui expliquent que, dans ces derniers pays, il y a peu de chances pour que des citadins réagissent favorablement à 1'annonce de la transformation de leur impasse résidentielle en voie traversante.

Loin d'interdire toute comparaison cependant, ces différences contextuelles permettent de mettre en évidence certains des présupposés des pratiques d'aménagement viaire actuellement en vigueur en Europe ou aux États-Unis. Elles offrent notamment la possibilité de montrer que la différenciation des rues est loin d'être pathologique. À condition qu'elle ne prenne pas la forme d'un fonctionnalisme extrême ${ }^{33}$, cette dernière doit même plutôt être considérée comme l'un des témoignages les plus clairs de la maturation de l'urbanisation. Elle est la réponse qu'apporte l'espace urbain au fait que, loin d'être simplement opposées, valeur territoriale et valeur locale de la rue entretiennent une relation complexe, où les forces de répulsion n'agissent qu'en concurrence avec des forces d'attraction. Ainsi, même si cela reste à confirmer en menant des enquêtes d'une plus grande envergure, il apparaît que, dans la capitale thaïlandaise, les couples aisés avec enfants privilégient les rues résidentielles désertes où leur progéniture peut s'ébattre sans danger, alors que les personnes à faible mobilité ou que les ménages modestes, soucieux de pouvoir ouvrir un commerce d'appoint, préfèrent les rues ou les quartiers plus animés et plus accessibles, quitte à parfois être dérangés par des bruits intempestifs. Pour ces personnes, les voies intermédiaires entre la rue résidentielle et le boulevard, ne sont pas aussi dépourvues d'attraits que de nombreux urbanistes voudraient le faire croire. Le constat peut paraître banal, mais au vu des pratiques actuelles d'aménagement viaire, il mérite d'être rappelé. 
1. Voir, entre autres : CETUR, Les enjeux des politiques de déplacement dans une stratégie urbaine, Bagneux, CETUR, 1994, p. 119-131.

2. D. Appleyard et al., Liveable Urban Streets : Managing Auto Traffic in Neighborhoods, Washington, US Department of Transportation, 1976, p. 31-77.

3. Idem, p. $60-70$.

4. C. L. Girling \& K. I. Helphand, Yard, Street, Park - The Design of Suburban Open Spaces, New York, John Wiley \& Sons, 1994, p. 60-61.

5. C. Buchanan et al., Traffic in Towns, A Study of the Long Term Problems of Traffic in Urban Areas, Londres, Report of the Sterring Group and Working Group appointed by the Minister of Transport, Her Majesty's Stationery Office, 1963, p. 47 (voir notamment la Figure 61).

6. Girling et Helphand, op. cit., p. 63

7. Idem, p. 103

8. La bibliographie sur ce type d'aménagement viaire est abondante. Voir, entre autres, L. Bonamoni, Le temps des rues - Vers un nouvel aménagement de l'espace rue, Lausanne, IREC, 1990, p. 15 $s q q$. Pour le détail de la filiation des pratiques urbanistiques évoquées ici, voir plus particulièrement, M. Southworth \& E. BenJoseph, Streets and the Shaping of Towns and Cities, New York, McGraw-Hill, 1997, p. 109 et s.

9. Onze fonctionnaires et cinq élus de municipalités de districts périphériques de l'agglomération de Bangkok ont été interrogés sur ces questions d'avril à juin 1996. D'autre part, les gérants d'une dizaine de lotissements ont été rencontrés au cours du $1^{\text {er }}$ semestre 1997

10. Ce soi est situé au nord-est de l'agglomération de Bangkok, dans le district de Lad Phrao. Parmi les quarante-neuf maisons de ville qui bordent le soi, quinze familles ont été rencontrées. Cinq des maisons étaient inoccupées au moment de l'enquête (fin 1996) ; les occupants de dix autres logements ont refusé de répondre. Enfin, dans les dix-neuf cas restants, les habitants étaient absents les jours où nous avons mené les entretiens.

11. D. Appleyard \& M. Lintell, Environmental Quality of City Streets, Working Paper $n^{\circ}$ 142, Berkeley, Univ. of Cal., 1970, p. 11.

12. D. Appleyard et al., op. cit., p. 53. Appleyard a d'ailleurs lui-même relevé des cas similaires à celui de la vendeuse de rue du soi Charœn Phon lors d'enquêtes que lui et son équipe ont réalisées à San Francisco (p. 42).

13. La sécurité, plus que toute autre valeur, reste au fondement de la plupart des aménagements viaires contemporains, ainsi que l'illustrent en France les publications du Centre d'études sur les réseaux, les transports, l'urbanisme et les constructions publiques (CERTU). Le programme «ville plus sûre, quartiers sans accidents » ou les opérations «zones 30 » sont orientés en priorité vers la modération de la vitesse et vers la réduction du risque d'accidents. Voir sur ce point les publications suivantes du CERTU : Ville plus sare, quartiers sans accidents: Savoir faire et techniques, 1990,318 p. ; Guide zone 30 - Méthodologie et recommandations, $1992,64 \mathrm{p}$.

14. D. Appleyard, « Streets Can Kill Cities: Third World Beware: Guidelines for Street Design in Third World Cities », Habitat International, vol. 7, n ${ }^{\circ} 3 / 4$, Londres, 1983, p. 120.

15. Pour l'urbanisme nord-américain, voir, outre les publications citées précédemment : A. Vernez-Moudon (ed.), Public Streets for Public Use, New York, Columbia Univ. Pr., 1991, 350 p. Cet ouvrage est d'ailleurs dédié à la mémoire de Donald Appleyard, décédé en 1982. Pour le cas européen, le guide d'aménagement viaire publié par Lydia Bonamoni (Le temps des rues) est particulièrement significatif. Les voies urbaines y sont classées en deux catégories les rues de quartiers et les axes principaux (voir notamment p. 15).
16. Voir à ce sujet A. Poungsomlee et H. Ross, Impact of Modernisation \& Urbanisation in Bangkok : an Integrative Ecological and Biosocial Study, Mahidol University, Australian National University, 1992, p. 37 sqq. ; R. Körff, Markets, Trade and State : Urbanism in Bangkok, University Bielefeld, 1992, p. 138.

17. Ces cas sont plutôt rares. La règle est plutôt que les lotissements restent fermés à tout trafic de transit. Cette situation ne tenant pas aux nuisances du trafic, mais à la peur de l'insécurité et aux caractéristiques du marché foncier, elle est ici volontairement passée sous silence, afin de ne pas alourdir l'exposé par des considérations trop éloignées des problèmes traités.

18. Ces informations ont été recueillies en mai 1997 au cours d'un entretien avec un membre du comité de copropriétaires du lotissement.

19. D. Appleyard \& M. Lintell, op. cit., p. 34-35.

20. Des observations semblables ont été faites ailleurs, notamment dans un soi voisin, le soi Rittikasem.

21. Voir notamment les observations sur ce point de $\mathrm{K}$. Lynch, Good City Form, MIT Press, 1994 (1 $1^{\text {re }}$ édition 1981), p. 264 ; P. Merlin, Géographie, économie et planification des transports, Paris, PUF, 1991, p. 282-283.

22. Girling et Helphand, op. cit., p. 171-211.

23. A. Faure, Entre les tours et les barres - Restructurer les espaces publics des grands ensembles, Lyon, CERTU, Délégation interministérielle à la ville, 1996, p 90.

24. Voir notamment à ce propos les recherches de Bernard Rouleau, qui montrent que, dans les arrondissements périphériques de Paris, les équipements liés à la rue se répartissent autour des carrefours importants, des axes radiaux et de quelques axes concentriques (Villages et faubourgs de l'ancien Paris, Histoire d'un espace urbain, Paris, Seuil, 1985, p. 33-36).

25. Pour les indicateurs de mobilité à Bangkok, voir les résultats de l'une des rares enquêtes transports menées dans cette ville, in : Japan International Cooperation Agency, The Study on Medium to Long Term Improvement/Management Plan of Road Transport in Bangkok, Main Report, Bangkok, 1990, p. 47-70. Les chiffres pour la France proviennent des enquêtes INSEE-INRETS 1993-1994.

26. P. Chalermpow Koanantakool, Urban Life and Urban People in Transition, Bangkok, TDRI, 1993, p. 50-52.

27. C. Goldblum, Métropoles de l'Asie du sud-est - Stratégies urbaines et politiques du logement, Paris, L'Harmattan, 1987, p. 123.

28. Quatre des sept commerçants interrogés très précisément (février 1997).

29. L. Couvreur, « La rue et les boutiques », in : P.H. Chombart de Lauwe et al., Paris et l'agglomération parisienne, Tome II, Méthodes de recherche pour l'étude d'une grande cité, Paris, PUF, 1952, p. 70. Voir aussi, Bernard Rouleau, Le tracé des rues de Paris, Paris, Presses du CNRS, 1988, p. 115.

30. V. Fouchier, Les densités urbaines et le développement durable - Le cas de l'Ile-de-France et des villes nouvelles, Paris, La documentation française, $1997,212 \mathrm{p}$

31. Girling et Helphand, op. cit., p. 171-211.

32. Pour un aperçu de l'impact de la notion de « qualité de vie » sur l'aménagement des espaces publics en France, voir : Plan urbain, Espaces publics, Paris, La documentation française, 1988, 132 p.

33. Pour une critique de la voie spécialisée telle qu'elle est conçue par les urbanistes dit « fonctionalistes», voir : J. L. Gourdon, "Deux formes urbaines : la rue et la voie spécialisée », séminaire Les transports et la ville, Paris, Ministère de l'Equipement, des transports et du logement, Ecole nationale des ponts et chaussées, séance du $1^{\text {er }}$ avril 1998, p. 51-59. 